\title{
Effect of Massage Therapy With and Without Elastic Bandaging on Pain, Edema, and Shoulder Dysfunction After Modified Radical Mastectomy: A Clinical Trial
}

\author{
Mehdi Khanbabayi Gol ${ }^{1}$, Davoud Aghamohamadi ${ }^{*}{ }^{\circledR}$
}

\begin{abstract}
Objectives: Pain and shoulder dysfunction are among the adverse and prevalent conditions in post-mastectomy women. Therefore, the present study aimed to examine massage therapy with and without elastic bandaging on pain, edema, and shoulder dysfunction after modified radical mastectomy.

Materials and Methods: This was a clinical trial conducted at Imam Reza hospital, Tabriz, Iran, from December 22, 2018, to May 5, 2019. Ninety women participated in this study and were randomly divided into three groups of 30 (i.e., Manual lymph drainage, Manual lymph drainage plus reduced-compression bandaging, and control) based on a random number table. Patients and their companions were instructed on the intervention to be performed at home on a daily basis 24 hours after surgery with the help of a trained research assistant. Data were entered in the relevant forms before and after the intervention, including demographic information, shoulder pain and dysfunction index (SPDI), and edema checklist. Data were analyzed through descriptive statistics, one-way ANOVA, and the Kruskal-Wallis test in SPSS 19. $P<0.05$ was considered statistically significant.

Results: The comparison of the main variables between the three groups before and after the intervention showed no statistically significant difference $(P>0.0560)$. The intervention could significantly change pain intensity and shoulder movement limitation $(P=0.001)$. However, there was no difference in the edema variable before and after the intervention $(P=0.25)$.

Conclusions: In general, massage therapy with and without elastic bandaging had a positive effect on shoulder movement limitation and pain whereas it had no such effect on edema drainage.

Keywords: Massage therapy, Elastic bandaging, Lymphedema, Mastectomy
\end{abstract}

\section{Introduction}

Lymphedema, with a postoperative incidence of $8 \%$ to $52 \%$ in the first 2 years, is an adverse complication of breast surgery, whose causes have not yet been well-established $(1,2)$. Lymphatic edema affects the upper limbs and, consequently, a person's physio-psychological life. Given the lack of definitive treatment for lymphatic edema in the current existing science, therapeutic measures aim to reduce the increased edema and maintain limb at the smallest size to the possible extent. Such treatments reduce the amount of accumulated fluid in the tissue and thus prevent lymphatic edema or infections (3). Upper limb lymphedema can lead to numerous complications such as increased limb volume, increased risk for infection, psychological complications, decreased local immunity in the affected side, movement limitation, decreased self-confidence, serious social and emotional problems, severe, sometimes uncontrollable pain, skin-associated alterations, and ultimately, problems in activities of daily living $(4,5)$.

Given the adverse effects of post-mastectomy lymphedema on various aspects of the treatment and life, it is imperative to manage this complication (5). Cancer-induced lymphedema is managed based on decongestant physiotherapy (6). In addition, manual lymph drainage and multilayer bandaging are the main therapeutic techniques for peripheral lymphedema (7). Although manual lymph drainage is an important part of lymphedema treatment, there is limited evidence for a clinical practice guideline (8). Various gentle massaging techniques are used to remove excess interstitial fluid and increase lymphatic transfer in manual lymph drainage (9). Bunce et al reported the positive effect of multidimensional massage therapy as a therapeutic intervention, pneumatic compression, bandaging, and self-care technique instruction on reducing postmastectomy lymphedema (10). Despite numerous studies regarding the positive effects of massaging and bandaging on lymphedema enhancement and control (11-13), some others have not reported any advantage of this type of therapy $(14,15)$. Therefore, the edema has specified points and the adverse effects such as pain and shoulder dysfunction in post-mastectomy women and surgical radical mastectomy can cause damage to the upper limb 
nerves and neuromuscular problems in the area, leading to these problems for several years after surgery (1618). Considering the above-mentioned explanations, the present study aimed to examine the effect of massage therapy with and without elastic bandaging on pain, edema, and shoulder dysfunction after modified radical mastectomy.

\section{Materials and Methods}

This clinical trial was conducted on female patients who underwent a modified radical mastectomy and were hospitalized at the Thoracic Surgery Unit of Imam Reza Hospital, Tabriz, Iran. A sample size of 30 cases for each group was determined based on a similar study by Norouzzadeh et al (19), and a total of 90 patients entered the study. Participants were selected from December 22, 2018, to May 5, 2019. Based on a random number table, they were randomly assigned to three groups of 30 , including the manual lymph drainage, the manual lymph drainage plus reduced-compression bandaging, and the control groups. The inclusion criteria were mastectomy with axillary lymph node dissection and the availability of a literate family member for massaging or bandaging. The exclusion criteria included a history of any type of surgery on the shoulder, as well as a history of previous lymphedema, shoulder dysfunction, and lymphatic system disorder.

\section{Instruments}

The data collection instruments consisted of a demographic information questionnaire, the Shoulder Pain and Dysfunction Index (SPADI), and an Edema Checklist. SPADI comprises two subscales of pain and shoulder useability assessment $(20,21)$. The pain assessment subscale included four items encompassing the ability to lie on the involved side, reach for something on a high shelf, touch the back of the neck, and push with the involved arm. After each intervention, patients were asked to mark their responses on each item of the visual analogue scale ranging from zero to ten representing no problem to the worst state, respectively (22). The shoulder dysfunction assessment encompassed eight items. Patients were asked to apply their ability in using the shoulder joint on a scale of $0-10$ ( 0 indicating no problem and 10 the greatest difficulty) for the following items: ability to wash the hair, reach the back, put on an undershirt or jumper, put on a shirt that buttons down the front, put on pants, place an object on a high shelf or rack, carry a heavy object of 10 pounds $(4.5 \mathrm{~kg})$, and pick something up from the back without turning. Arm edema was measured in two steps as circumferential and volumetric measurements. First, the limb circumference was measured using a tape measure in predetermined areas including the metacarpophalangeal joint, the first web space, the wrist joint, $15 \mathrm{~cm}$ below the elbow, and 7.5, 15, and $22.5 \mathrm{~cm}$ above the elbow in the elbow area. Patients assumed a sitting position with their elbows held straight and measurement was carried out on a single day for both sides. Then, the limb volume was measured using a volumeter through fluid transfer. For this measurement, patients were asked to drop their limbs slowly in the volumeter until the horizontal bar at its end was placed between the space of the second and third fingers. The transferred fluid rate was measured and the same procedure was repeated for the healthy side. Therefore, the volumes of both arms were measured, allowing for the difference between the transferred fluid in both arms, as well as the calculation of the volume of lymphedema in the involved limb.

\section{The Intervention Process}

For accurate interventions, a nurse who was a member of the research team learned the proper massaging technique and reduced-compression bandaging after 6 months of training under the supervision of the physiotherapy team of Tabriz University of Medical Sciences. Twenty-four hours after surgery and before the discharge, a training session was held for patients and one family member who always lived with them. The "manual lymph drainage" group was instructed on the proper massaging technique, and the "manual lymph drainage plus reduced-compression bandaging" group received instruction on the proper massaging and bandaging techniques. In addition to inperson instruction and practice, an instructional pictorial pamphlet with adequate descriptions was handed to patients and their companions in order to achieve greater accuracy in massaging and bandaging. In the "manual lymph drainage" group, the intervention was performed by instructing patients and their companions to massage the area each day for 20 minutes. This process was continued for one month. The manual lymph drainage method was based on the Vodder's technique. In addition, instructions on massaging with the specified technique by the patient are summarized as follows: Drawing 5 diaphragmatic breaths, massaging from under the auricle to the bottom of the neck, from the medullary cavity of the exterior clavicle to the interior, and from the side of surgery to the opposite arm's armpit and the armpit itself (23). The "manual lymph drainage plus reduced-compression bandaging" group received intervention in the form of elastic reduced-compression bandages wrapped around the limb on the side of the surgery in addition to massage therapy. Patients were instructed to keep the bandage around the limb on the side of the surgery for at least 6 hours a day (preferably during nighttime sleep or after each massage session at the patient's discretion). This process was continued for one month. In this bandaging technique, small bandages (Mollelast) would be first wrapped around the fingers. After covering the wrist to arm with an openweave tubular bandage and Webril cotton bands, reducedcompression bandages would be placed in two layers on the wrist to the elbow and the elbow to the arm. Pain intensity and shoulder dysfunction were assessed on the 
seventh day after operation in order to compare the preand post-intervention states given the extent of radical mastectomy, the associated pain with the incision area, and postoperative hematoma-induced edema. Further, the edemas were measured and recorded by the research nurse on two occasions, namely, 24 hours after the operation and one month after the initiation of intervention. In both intervention groups, the patient and a family member were asked to fill out the requested SPADI items of the checklist and questionnaire. The specified interventions were not performed for the control group although they received routine post-mastectomy interventions. The research nurse's contact number was provided to patients for follow-up regarding correct and timely interventions. Furthermore, interventions by patients or family members were regularly monitored in person or via phone calls. If required, necessary instructions would be given to patients upon visiting the oncology clinic. At the end of the onemonth period, the questionnaires and checklists were collected by the research nurse. Eventually. The data were analyzed using descriptive statistics, one-way ANOVA, and the Kruskal-Wallis test in SPSS 19. The $P<0.05$ was considered statistically significant.

\section{Results}

Demographic Findings

The results of this section indicated that the mean age of the patients was $44.12 \pm 6.09,43.29 \pm 6.55$, and $45.81 \pm 7.01$ years in the massage, massage plus bandage, and the control groups, respectively. Additionally, 18 (60\%), 16 (52.23\%), and $15(50 \%)$ patients from the massage, massage plus bandage, and control groups undergo surgery on the left side of the body, respectively. There was no statistically significant difference between the hand circumference, pain intensity, and shoulder movement limitation of the three groups before intervention (Table 1).

Findings for Hand Circumference

Given the normality of the random sample in the dependent variable (mean hand circumference) based on the Kolmogorov-Smirnov test $(P>0.05)$, the parametric one-way ANOVA test was used to compare the mean post-intervention hand circumferences on the side of operation between the three groups (control, massage, and massage plus bandage). According to the results, there was no significant reduction in the mean hand circumference difference $(\mathrm{cm})$ between the three groups on the 30th day $(P=0.255)$, the details of which are provided in Table 2 .

\section{Findings for Pain Assessment}

Given the abnormality of the dependent variable of pain intensity $(P<0.05)$, the non-parametric Kruskal-Wallis test was utilized to compare the mean post-intervention pain intensity (after 30 days) between the three groups (control, massage, and massage plus bandage). Based on the results (Table 2), a significant reduction was observed in the mean pain intensity difference between the three groups on the 30th day $(P=0.001)$.

\section{Findings for Shoulder Movement Limitation}

Regarding the abnormalities of the musculoskeletal system of the upper extremity $(P<0.05)$, a nonparametric KruskalWallis statistical test was used at the end of the intervention (30th day) between the three groups (control, massage, massage + bandage). The results showed a significant decrease compared to the pre-intervention group in the

Table 1. Comparison of Hand Circumference, Pain Intensity, and Upper Limb Musculoskeletal System Tests Before Intervention

\begin{tabular}{|c|c|c|c|c|}
\hline & $\begin{array}{c}\text { Massage }(n=30) \\
\text { Mean } \pm \text { SD }\end{array}$ & $\begin{array}{l}\text { Massage Plus Bandage }(n=30) \\
\text { Mean } \pm S D\end{array}$ & $\begin{array}{l}\text { Control }(n=30) \\
\text { Mean } \pm \text { SD }\end{array}$ & $P$ value \\
\hline Edema & $23.12 \pm 3.21$ & $22.55 \pm 2.85$ & $22.19 \pm 2.91$ & 0.711 \\
\hline Pain intensity & $4.25 \pm 1.65$ & $4.15 \pm 1.15$ & $4.51 \pm 1.11$ & 0.851 \\
\hline Empty can, No. (\%) & $8(26.66)$ & $9(30)$ & $9(30)$ & 0.715 \\
\hline Neer impingement, No. (\%) & $9(30)$ & $8(26.66)$ & $7(23.33)$ & 0.711 \\
\hline Hawkins Kennedy impingement, No. (\%) & $5(16.66)$ & $5(16.66)$ & $4(13.33)$ & 0.803 \\
\hline Yergason, No. (\%) & $6(20)$ & $5(16.66)$ & $6(20)$ & 0.901 \\
\hline Compression, No. (\%) & $9(30)$ & $8(26.66)$ & $9(30)$ & 0.811 \\
\hline Cross over, No. (\%) & $6(20)$ & $5(16.66)$ & $6(20)$ & 0.903 \\
\hline Resistive tennis elbow, No. (\%) & $3(10)$ & $3(10)$ & $4(13.33)$ & 0.890 \\
\hline Cozens, No. (\%) & $5(16.66)$ & $6(20)$ & $5(16.66)$ & 0.889 \\
\hline Passive tennis elbow, No. (\%) & $9(30)$ & $10(33.33)$ & $8(26.66)$ & 0.703 \\
\hline Phalen test, No. (\%) & $3(10)$ & $4(13.33)$ & $3(10)$ & 0.515 \\
\hline Revers Phalen, No. (\%) & $6(20)$ & $7(23.33)$ & $8(26.66)$ & 0.690 \\
\hline Tinel's sign, No. (\%) & $3(10)$ & $3(10)$ & $5(16.66)$ & 0.503 \\
\hline Pronator teres, No. (\%) & $5(13.33)$ & $5(13.33)$ & $3(10)$ & 0.609 \\
\hline Finkelestein, No. (\%) & $11(36.66)$ & $10(33.33)$ & $12(40)$ & 0.706 \\
\hline Resisted isometric movement, No. (\%) & $3(10)$ & $3(10)$ & $2(06.66)$ & 0.801 \\
\hline
\end{tabular}

Note. SD: Standard deviation. 
Table 2. Comparison of Hand Circumference, Pain Intensity, and Upper Limb Musculoskeletal System Tests After Intervention

\begin{tabular}{lcccc}
\hline & Massage $(\mathbf{n}=\mathbf{3 0})$ & Massage Plus Bandage $(\mathbf{n}=\mathbf{3 0})$ & Control $(\mathbf{n}=\mathbf{3 0})$ & \multirow{2}{*}{$\boldsymbol{P}$ Value } \\
\cline { 2 - 4 } & Mean \pm SD & Mean \pm SD & Mean \pm SD & 0.225 \\
\hline Edema & $25.25 \pm 3.78$ & $24.12 \pm 3.12$ & $25.59 \pm 3.18$ & $3.81 \pm 1.60$ \\
Pain intensity & $2.11 \pm 1.39$ & $2.19 \pm 1.25$ & $10(33.33)$ & 0.001 \\
Empty can, No. (\%) & $5(16.66)$ & $5(16.66)$ & $9(30)$ & 0.011 \\
Neer impingement, No. (\%) & $7(23.33)$ & $5(16.66)$ & $6(30)$ & 0.001 \\
Hawkins Kennedy impingement, No. (\%) & $1(03.33)$ & $0(0)$ & $8(26.66)$ & 0.001 \\
Yorgason, No. (\%) & $2(06.66)$ & $1(03.33)$ & $11(36.66)$ & 0.003 \\
Compression, No. (\%) & $3(10)$ & $2(06.66)$ & $8(26.66)$ & 0.002 \\
Cross over, No. (\%) & $1(03.33)$ & $1(03.33)$ & $5(16.66)$ & 0.001 \\
Resistive tennis elbow, No. (\%) & $0(0)$ & $0(0)$ & $9(30)$ & 0.001 \\
Cozens, No. (\%) & $1(03.33)$ & $0(0)$ & $12(40)$ & 0.001 \\
Passive tennis elbow, No. (\%) & $2(06.66)$ & $2(06.66)$ & $5(16.66)$ & 0.001 \\
Phalen test, No. (\%) & $0(0)$ & $0(0)$ & $9(30)$ & 0.003 \\
Revers Phalen, No. (\%) & $1(03.33)$ & $1(03.33)$ & $15(50)$ & $0 / 001$ \\
Tinel's sign, No. (\%) & $0(0)$ & $0(0)$ & $6(20)$ & 0.001 \\
Pronator teres, No. (\%) & $1(03.33)$ & $0(0)$ & $15(50)$ & 0.006 \\
Finkelestein, No. (\%) & $6(20)$ & $2(06.66)$ & $0(0)$ & $9(30)$ \\
Resisted isometric movement, No. (\%) & $0(0)$ & & & 0.006 \\
\hline
\end{tabular}

Note. SD: Standard deviation.

three groups on day 30 so that the pathological variables of the shoulder $(P=0.001)$, tennis elbow $(P=0.001)$, carpal tunnel syndrome $(P=0.001)$, and De Quervain's tenosynovitis $(P=0.001)$ were significantly associated with improvement.

\section{Discussion}

The present study aimed to examine massage therapy with and without elastic bandaging on pain, edema, and shoulder dysfunction after modified radical mastectomy. The results indicated that massage therapy with and without elastic bandaging had a positive effect on pain intensity and movement limitation whereas it did not contribute to edema drainage. The results of studies on the effects of massage therapy on edema reduction conducted in Iran suggested a decrease in edema volume compared to the control groups $(30,31)$. In another study, Ghaderi et al reported that bandaging can result in lymph drainage through pressure on the tissue. Contrary to the present research, positive results were observed from massage therapy on decreased edema in other studies $(32,33)$. Similarly, Karafa et al found the positive effects of elastic bandage on the reduction of lymphedema (34). In addition, Butt et al demonstrated the positive effects of bandage plus exercise on lymph reduction (35), which contradicts the findings of the current study.

Likewise, in numerous studies, bandaging and massage therapy have been reported to have beneficial effects on pain relief in patients with lymphedema $(11,36)$. With the existing technique that alleviates nerves in the edematous area, massage therapy with and without elastic bandage appears to relieve pain.

Other studies, such as the study by Kim et al obtained similar results regarding improving the condition of the nervous system in the shoulder region after the massage therapy. Therapeutic massage seems to improve the function of the nerve of the shoulder region by creating relaxation in the shoulder region and having positive effects on the nerves. Massage therapies also release normal analgesics in the body and greatly reduces pain in that area (37).

Shoulder movement limitation in both intervention groups was lower than the control group, indicating the efficacy of the intervention in shoulder movement. In similar studies, massage therapy with and without elastic bandaging also had positive effects on shoulder movement $(19,30,36)$.

\section{Conclusions}

In general, massage therapy with and without elastic bandaging demonstrated positive effects on shoulder movement limitation and pain whereas it did not contribute to edema drainage.

\section{Limitations of the Study}

The limitations or disadvantages of the study included the short duration of the study and the lack of attention to such treatments as radiotherapy or chemotherapy.

\section{Suggestions for Future Studies}

Further studies are recommended with an emphasis on removing the specified limitations.

\section{Conflict of Interests}

Authors declare that they have no conflict of interests. 


\section{Ethical Issues}

The observed ethical considerations in this study were similar to those of other studies (24-29), the most notable of which included obtaining an ethical code from the Regional Medical Ethics Committee (Ethics no. IR.TBZMED.REC.1397.1059) and registering the study in the Iranian Registry of Clinical Trials (identifier: IRCT20120605009948N6; https://www.irct.ir/ trial/38485). Moreover, informed consent was obtained and the research objectives were explained in plain and comprehensible language to the participants, and they were ensured of voluntary participation in the study and were offered free-of-charge physiotherapy and bandaging services.

\section{Financial Support}

This study was granted by Tabriz University of Medical Sciences..

\section{Acknowledgments}

The present paper is part of a research project approved by the Clinical Research Development Unit, Shohada hospital of Tabriz University of Medical Sciences. The researchers would like to give their gratitude to the Research Center and the Health Vice-chancellor of Tabriz University of Medical Sciences for financial support in the study.

\section{References}

1. Paskett ED, Naughton MJ, McCoy TP, Case LD, Abbott JM. The epidemiology of arm and hand swelling in premenopausal breast cancer survivors. Cancer Epidemiol Biomarkers Prev. 2007;16(4):775-782. doi:10.1158/10559965.epi-06-0168

2. Wanchai A, Armer JM, Stewart BR, Lasinski BB. Breast cancer-related lymphedema: a literature review for clinical practice. Int J Nurs Sci. 2016;3(2):202-207. doi:10.1016/j. ijnss.2016.04.006

3. Poole K, Fallowfield LJ. The psychological impact of postoperative arm morbidity following axillary surgery for breast cancer: a critical review. Breast. 2002;11(1):81-87. doi:10.1054/brst.2001.0369

4. Türk G, Khorshid L. The complete decongestive therapy in lymphedema management developing in relation with mastectomy. J Breast Health. 2011;7(2):96-100.

5. Hosseini Bereshneh A, Soltani D, Veisi N, Khademi M, Modarressi $\mathrm{MH}$. Horizons of personalized medicine in breast cancer treatment: review article. Tehran University Medical Journal. 2016;74(9):607-613. [Persian].

6. Arrault M, Vignes S. [Management of lymphedema of the upper extremity after treatment of breast cancer]. Bull Cancer. 2007;94(7):669-674. doi:10.1684/bdc.2007.0400

7. Leduc O, Crasset V, Leleu C, et al. Impact of manual lymphatic drainage on hemodynamic parameters in patients with heart failure and lower limb edema. Lymphology. 2011;44(1):13-20.

8. Williams A. Manual lymphatic drainage: exploring the history and evidence base. Br J Community Nurs. 2010;15(Suppl 3):S18-24. doi:10.12968/bjcn.2010.15.
Sup3.47365

9. Moseley AL, Carati CJ, Piller NB. A systematic review of common conservative therapies for arm lymphoedema secondary to breast cancer treatment. Ann Oncol. 2007;18(4):639-646. doi:10.1093/annonc/mdl182

10. Bunce IH, Mirolo BR, Hennessy JM, Ward LC, Jones LC. Post-mastectomy lymphoedema treatment and measurement. Med J Aust. 1994;161(2):125-128. doi:10.5694/j.1326-5377.1994.tb127342.x

11. Mondry TE, Riffenburgh RH, Johnstone PA. Prospective trial of complete decongestive therapy for upper extremity lymphedema after breast cancer therapy. Cancer J. 2004;10(1):42-48. doi:10.1097/00130404-200401000-00009

12. Johansson K, Lie E, Ekdahl C, Lindfeldt J. A randomized study comparing manual lymph drainage with sequential pneumatic compression for treatment of postoperative arm lymphedema. Lymphology. 1998;31(2):56-64.

13. Ochałek K, Gradalski T. Manual lymph drainage may not be a necessary component in lymphedema treatment. J Pain Symptom Manage. 2010;39(5):e1-2. doi:10.1016/j. jpainsymman.2010.01.008

14. Andersen L, Højris I, Erlandsen M, Andersen J. Treatment of breast-cancer-related lymphedema with or without manual lymphatic drainage--a randomized study. Acta Oncol. 2000;39(3):399-405. doi:10.1080/028418600750013186

15. Mohammadpour $M$, Yaseri $M$, Mahmoudi $M$, Entezar Mahdi R. Estimation of Survival in Women Diagnosed with Breast Cancer with Cure Survival in West-Azerbaijan and East-Azerbaijan Provinces. Journal of School of Public Health and Institute of Public Health Research. 2018;16(1):63-74. [Persian].

16. Liao LR, Huang M, Lam FM, Pang MY. Effects of wholebody vibration therapy on body functions and structures, activity, and participation poststroke: a systematic review. Phys Ther. 2014;94(9):1232-1251. doi:10.2522/ptj.20130366

17. Xu X, McGorry RW, Lin JH. A regression model predicting isometric shoulder muscle activities from arm postures and shoulder joint moments. J Electromyogr Kinesiol. 2014;24(3):419-429. doi:10.1016/j.jelekin.2014.02.004

18. Jung DE, Moon DC. The effects of shoulder joint abduction angles on the muscle activity of the serratus anterior muscle and the upper trapezius muscle while vibrations are applied. J Phys Ther Sci. 2015;27(1):117-119. doi:10.1589/ jpts.27.117

19. Norouzzadeh R, Heidari M, Sedighiani A, Kiani-Asiabar A, Faghihzadeh S. Comparative effects of massage therapy and bandage on shoulder pain, edema and dysfunction after the modified radical mastectomy. Feyz Journal of Kashan University of Medical Sciences. 2012;16(3):205211. [Persian].

20. Paul A, Lewis M, Shadforth MF, Croft PR, Van Der Windt DA, Hay EM. A comparison of four shoulderspecific questionnaires in primary care. Ann Rheum Dis. 2004;63(10):1293-1299. doi:10.1136/ard.2003.012088

21. Ekeberg OM, Bautz-Holter E, Tveitå EK, Keller A, Juel NG, Brox JI. Agreement, reliability and validity in 3 shoulder questionnaires in patients with rotator cuff disease. BMC Musculoskelet Disord. 2008;9:68. doi:10.1186/1471-24749-68

22. Hayes SC, Johansson K, Stout NL, et al. Upper-body 
morbidity after breast cancer: incidence and evidence for evaluation, prevention, and management within a prospective surveillance model of care. Cancer. 2012;118(8 Suppl):2237-2249. doi:10.1002/cncr.27467

23. Williams A. Patient self-massage for breast cancer-related lymphoedema. J Community Nurs. 2006;20(6):24-29.

24. Hosseinzadeh $\mathrm{H}$, Golzari SE, Abravesh M, et al. Effect of low dose dopamine on early graft function in living unrelated kidney donors. Urol J. 2012;9(1):389-396. doi:10.22037/ uj.v9i1.1387

25. Aghamohammadi D, Eidi M, Lotfi A, et al. Effect of low level laser application at the end of surgery to reduce pain after tonsillectomy in adults. J Lasers Med Sci. 2013;4(2):7985.

26. Vahedi P, Salehpour F, Aghamohammadi D, et al. Single dose preemptive amitriptyline reduces postoperative neuropathic pain after lumbar laminectomy and discectomy: a randomized placebo-controlled clinical trial. Neurosurg Q. 2010;20(3):151-158. doi:10.1097/ WNQ.0b013e3181ebce15

27. Zomorrodi A, Mohammadipoor Anvari H, Kakaei F, Solymanzadeh F, Khanlari E, Bagheri A. Bolus Injection Versus Infusion of Furosemide in Kidney Transplantation: A Randomized Clinical Trial. Urol J. 2017;14(2):3013-3017. doi:10.22037/uj.v14i2.3787

28. Aghamohammadi D, Gargari RM, Fakhari S, Bilehjani E, Poorsadegh S. Classic versus inguinal approach for obturator nerve block in transurethral resection of bladder cancer under spinal anesthesia: a randomized controlled trial. Iran J Med Sci. 2018;43(1):75-80.

29. Naghipour B, Faridaalaee G, Shadvar K, Bilehjani E, Khabaz AH, Fakhari S. Effect of prophylaxis of magnesium sulfate for reduction of postcardiac surgery arrhythmia: Randomized clinical trial. Ann Card Anaesth. 2016;19(4):662-667. doi:10.4103/0971-9784.191577.

30. Ghaderi F, Amiri SN, Aghazade Z. Comparative study between two treatment methods on post mastectomy lymphadema reduction: standard method and complete decongestive therapy. Urmia Medical Journal. 2012;23(1):47-51. [Persian].

31. Khoshnazr T, Mohammadi F, khankeh H, Haghighat S. The Effect of Rehabilitation Program on Lymphedema- related Arm Pain, and Volume after Mastectomy. Iran Journal of Nursing. 2011;24(70):72-81. [Persian].

32. Alande AA, Sagar JH, Shinde SB. Effect of Early Physiotherapy in Post Operative Radical Mastectomy for Lymphedema. Indian J Physiother Occup Ther. 2017;11(2):190-194. doi:10.5958/0973-5674.2017.00059.4

33. Zamani S, Hagigat S, Okhovatian F. Physical Therapy Approach in Shoulder Impairment Along With Lymphedemea after Breast Cancer Surgery: A Case Study. J Clin Physiother Res. 2017;2(3):145-151. doi:10.22037/jcpr. v2i3.17098

34. Karafa M, Karafova A, Szuba A. The effect of different compression pressure in therapy of secondary upper extremity lymphedema in women after breast cancer surgery. Lymphology. 2018;51(1):28-37.

35. Butt A, Ijaz Gondal M, Qamar M. Effectiveness of compression therapy along with exercise to reduce upper limb lymphedema in breast cancer patients. Saudi J Sports Med. 2017;17(3):148-152.doi:10.4103/sjsm.sjsm_23_17

36. Forchuk C, Baruth P, Prendergast M, et al. Postoperative arm massage: a support for women with lymph node dissection. Cancer Nurs. 2004;27(1):25-33. doi:10.1097/00002820200401000-00004

37. Kim M, Lee M, Kim M, Oh S, Jung S, Yoon B. Effectiveness of therapeutic inflatable ball self-exercises for improving shoulder function and quality of life in breast cancer survivors after sentinel lymph node dissection. Support Care Cancer. 2019;27(7):2349-2360. doi:10.1007/s00520019-4656-0

(c) 2020 The Author (s); This is an open-access article distributed under the terms of the Creative Commons Attribution License (http://creativecommons.org/licenses/by/4.0), which permits unrestricted use, distribution, and reproduction in any medium, provided the original work is properly cited. 